\title{
Malpasset, la seule rupture totale d'un barrage-voûte
}

\section{P. DUFFAUT}

130, rue de Rennes

75006 Paris

pierre.duffaut@dbmail.com
Pour régulariser l'alimentation en eau des communes du littoral, le département du Var s'est doté d'un barrage au lieudit Malpasset, un barrage-voûte construit en 1952-1954 sur le Reyran, dont le plein remplissage a été différé en raison des retards d'une expropriation. Lors de l'automne très pluvieux de 1959, l'ouvrage a été ruiné, et le flot libéré a causé plus de $\mathbf{4 0 0}$ morts et des dégâts considérables dans la basse vallée et la ville de Fréjus. Comme aucun barragevoûte n'avait connu de rupture auparavant, et comme la rupture avait manifestement affecté le terrain de fondation, la catastrophe a suscité un ensemble exceptionnel de calculs et surtout d'essais pour remonter aux causes de la rupture, ce qui a ouvert des chapitres nouveaux dans la connaissance scientifique des massifs rocheux, et permis d'émettre des recommandations pour l'avenir. Ĺapplication de la mécanique des roches au génie civil a pris corps dans les années qui ont suivi.

Mots-clés : barrage, mécanique des roches, gneiss, faille, sous-pression, essais sur site.

\section{Malpasset, the only total failure of an arch dam}

In order to regulate the water supply for its coastal communities, the Var department equipped itself with a reservoir dam at Malpasset site. An arch dam was built in $1952-$ 1954 on the Reyran river. The filling up was delayed due to an expropriation procedure. During the very rainy autumn 1959, the dam failed and the flow made more than 400 casualties and huge damages along the lower valley and in the Fréjus city. As no failure of arch dams had been reported before, and as the failure obviously took place within the foundation ground, the catastrophe aroused exceptional analyses and testing in order to get back to the causes of failure. So, new fields have opened in the scientific knowledge of rock masses and recommendations have been issued for the future. Within the years after, rock mechanics began to better apply to civil engineering.

Key words: dam, rock mechanics, gneiss, fault, uplift, in situ tests. 


\section{Introduction}

Les besoins d'eau potable et d'irrigation de la côte varoise ont conduit le département du Var à projeter et construire un barrage au lieudit Malpasset, sur la vallée du Reyran, petit fleuve côtier qui traverse la basse ville de Fréjus. L'étroitesse de la vallée se prêtait à un barrage-voûte de $60 \mathrm{~m}$ de haut, appuyé sur un terrain cristallin (gneiss parcouru de filons de pegmatite). Il a été construit entre 1952 et 1954, mais son remplissage complet a été différé en raison d'une concession minière non expropriée à l'amont. La pluviosité exceptionnelle de l'automne 1959 a entraîné un remplissage rapide des derniers mètres sous le seuil du déversoir de crue, qui n'a pas été tout à fait atteint. L'exploitant ${ }^{1}$ avait laissé dépasser la cote de retenue autorisée, afin de préserver le chantier du pont de l'autoroute en construction à l'aval. Le soir du 2 décembre 1959 la voûte a été emportée et la vallée dévastée jusqu'à la mer.

Après la description du barrage et le rappel des étapes de sa construction et de son exploitation, l'article présente les constatations sur le terrain : les éléments restés debout en rive droite et la crevasse au pied amont qui atteste leur déplacement; le dièdre ouvert au sein du versant rive gauche à l'aval immédiat des parties de la voûte disparues (on comprendra plus tard que la partie basse de la voûte en rive gauche a été emportée la première, avant la ruine complète de l'ouvrage). La qualité du béton n'était pas en cause et au contraire les blocs transportés à l'aval montraient que la rupture s'était produite au sein du rocher de fondation. Ensuite sont détaillées les principales étapes des investigations et des recherches de mécanique des roches : les études de laboratoire, effectuées au Laboratoire de mécanique des solides de l'École polytechnique traitées dans l'article suivant (Habib, 2010, p. 19) mettent en évidence une fissuration matricielle très importante qui se traduit par une grande dispersion des résistances et un effet d'échelle considérable (Bernaix, 1967), ainsi que par une sensibilité extraordinaire de la perméabilité à la contrainte ; les mesures sur le terrain réalisées par une équipe d'Électricité de France montrent pour leur part une déformabilité plus grande que celles d'autres fondations de barrages français qui ont pu leur être comparées (Groupe de travail CFGB, 1967). La perméabilité naturellement faible de la roche est donc considérablement diminuée dans la zone où

(1) La direction départementale du Génie rural, agissant pour le compte du propriétaire, le conseil général du Var. s'exerce la poussée de la voûte, alors que la déformabilité entraîne l'ouverture d'une crevasse au pied amont. Associée à une faille préexistante à l'aval, cette crevasse aurait individualisé le dièdre rive gauche, et fortement aggravé la poussée hydrostatique sur l'ensemble barrage et fondation.

L'ensemble des réflexions et travaux engagés en France et dans le monde entier après la ruine de ce barrage a donné un essor nouveau à la mécanique des roches, reconnue désormais comme une discipline à part entière.

\section{2}

\section{Présentation du barrage et de sa rupture}

\section{7}

\section{Description sommaire du barrage dans son site}

Le département du Var a fait construire le barrage de Malpasset afin d'assurer l'alimentation en eau de la zone côtière, à la fois pour les usages urbains de Fréjus, Saint-Raphaël, Sainte-Maxime et Saint-Tropez, dont la population était en forte augmentation, et pour l'irrigation des cultures dans la plaine alluviale de l'Argens. En effet, le climat local comporte d'importantes sécheresses estivales et des pluies d'automne parfois très abondantes. La vallée du Reyran pouvait constituer une assez vaste cuvette de retenue inhabitée à l'amont d'un défilé plus étroit, propre à stocker 55 millions de mètres cubes derrière un barrage relativement modeste. Le terrain cristallin du massif du Tanneron (appartenant à l'Estérel) qui constitue les deux versants de ce défilé a été jugé apte à supporter un barrage alors que la retenue baigne pour partie ces mêmes terrains ainsi qu'un synclinal sédimentaire d'âge carbonifère. Toutes ces roches sont considérées comme imperméables.

Le barrage (Figs. 1 et 2) était une voûte mince à double courbure, d'un modèle courant à l'époque en France $^{2}$; son couronnement était à la cote 102,55 NGF, le lit du Reyran à la cote 42 , la ( retenue normale ) à 98,50 (le tableau I rassemble les principales mesures). L'arc supérieur était appuyé en rive gauche sur

(2) Et dans le monde : l'ouvrage de Mary (1967) estime à 600 le nombre des barrages-vouites alors en service.

Tasisaul Principales mesures du barage de Malpasset et de sa retenue. Main figures of Malpasset dam and reservoir.

\begin{tabular}{l|l|l}
\hline Hauteur sur fondation & $65 \mathrm{~m}$ & $60 \mathrm{~m}$ sur le lit du ruisseau \\
Longueur en crête & $223 \mathrm{~m}$ & plus 22 m de la culée rive gauche \\
Rayon amont en crête & $105 \mathrm{~m}$ & angle au centre $135 \mathrm{~g}(121,5 \mathrm{degrés})$ \\
Épaisseur maximale & $9 \mathrm{~m}$ environ & $6,9 \mathrm{~m}$ au pied de la console centrale \\
Dimensions de la culée & $4: 22 \mathrm{~m}, 1: 6,5 \mathrm{~m}, \mathrm{~h}: 10 \mathrm{~m}$ & \\
Volume de béton & $5000 \mathrm{~m}^{3}$ & $47 \mathrm{hm}^{3}$ à la cote de retenue normale \\
Capacité de la retenue & $55000000 \mathrm{~m}^{3}$ & \\
\hline
\end{tabular}




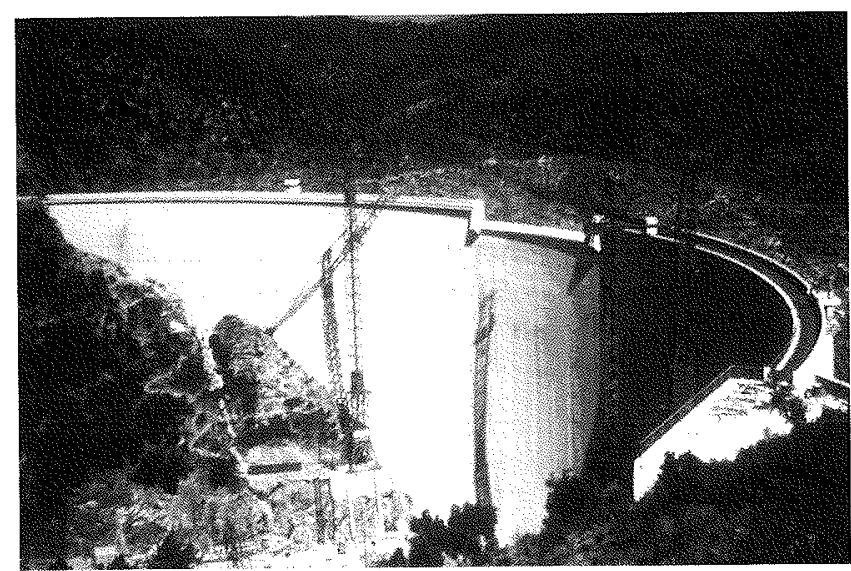

16. 1

Photographie du barrage en fin de construction, montrant la culée rive gauche et le seuil déversant; les grues qui ont assuré le bétonnage sont encore en place, mais le tapis de réception du jet n'est pas encore construit (photo Coyne et Bellier). Photo of the dam at end of construction, showing the left bank thrust block and the overflow sill the cranes used for pouring the concrete are yet standing, the apron receiving the overflow is not yet built (photo Coyne et Bellier)

une culée massive, assortie d'un « mur en aile » pour lui éviter de subir la poussée de l'eau. La coque a été construite en 16 plots verticaux indépendants, séparés par 15 joints radiaux (Fig. 3 , lettres de $A$ à $O$; le joint $P$ la sépare de la culée, partagée en deux par le joint Q).

Un seuil déversant (2) 3 long de 29,7 m à la cote 100,40 échancrait le couronnement, et le terrain au pied aval était protégé de l'impact de la lame d'eau déversante par un tapis de béton (4) ancré et drainé. Le couronnement était muni d'éléments préfabriqués formant un passage entre deux garde-corps (passage limité sur chaque rive au seuil déversant). Une conduite de vidange munie d'une vanne papillon traversait le béton à la cote 46,25 (sur un socle épais de $20,45 \mathrm{~m}$ ) et la prise d'eau était aménagée en rive gauche à la cote 79,5 suivie d'un organe de réglage du débit et d'une conduite en béton vers l'aval. Pendant la construction, un élargissement à la base du joint $\mathrm{H}$ a permis le passage du

(3) Ces chiffres entre parenthèses renvoient à ceux de la figure 2. débit du ruisseau, évitant le recours à une galerie de dérivation; cet orifice a été fermé après la mise en service de la vidange de fond.

Le barrage a noyé le chemin qui suivait le fond de la vallée (rive droite), chemin hérité d'une voie ferrée d'intérêt local, abandonnée de longue date, ainsi que les restes de l'aqueduc romain de Fréjus, qu'on devine à mi-hauteur sur la figure 6 .

\section{2.}

\section{Étapes du projet, de la construction, de l'exploitation}

Le site avait été envisagé de longue date et après des études préliminaires, dès 1946, le choix d'un barrage-voûte a été arrêté, puis le marché de construction signé le 25 février 1952 ; les terrassements ont commencé en avril, les premiers bétons étant coulés en août. Le barrage est achevé en avril 1954, et le tapis de réception des déversés est bétonné en août et septembre. En quelques zones, et notamment sous la culée, l'état d'altération du rocher a conduit à approfondir les fouilles avant de couler le béton. La liaison bétonrocher a été traitée par injection de coulis de ciment; un voile d'injection profond a été jugé inutile du fait de la très faible perméabilité du terrain.

On notera que la surveillance du comportement de l'ouvrage pendant les années de son premier remplissage était très sommaire : la déformation de la voûte est mesurée chaque été par une société de topographie (Figs. 3 et 4), et un gardien logé à proximité effectue des tournées d'inspection visuelle (il a ainsi signalé en novembre une venue d'eau dans le versant rive droite et l'apparition de fissures du tapis, également côté rive droite) ; il est chargé aussi du petit entretien et, à l'occasion, des manœuvres de vannes.

Le remplissage a débuté le 20 avril 1954, à la fermeture du joint élargi, et les pluies de l'automne ont fait monter le niveau jusqu'à la cote 80 , ce qui assure l'usage de la conduite de distribution d'eau. Il est resté stabilisé en 1955 en raison du retard des procédures d'expropriation déjà citées, et des paliers successifs sont observés ensuite, aux environs des cotes 83-85 en 1956-1957, 87,5 en 1958, puis 93 pendant l'été 1959. Les pluies exception-
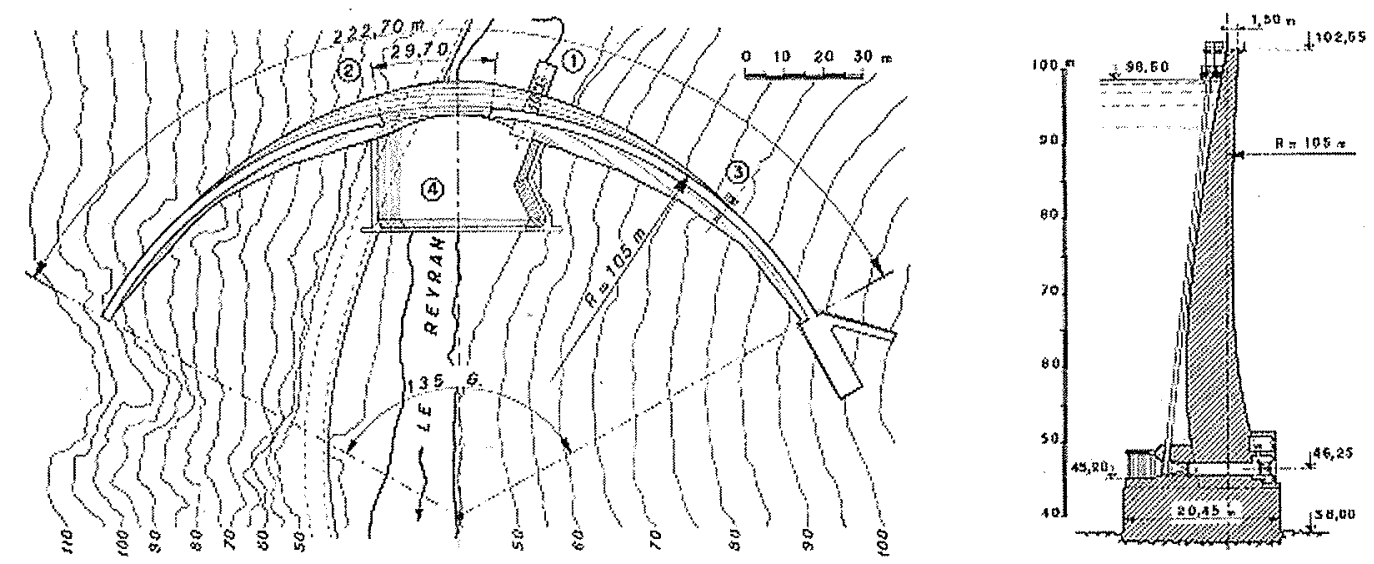

1G. 2 Vue en plan et coupe verticale sur la vidange de fond; le mur en aile évite la poussée de l'eau sur la culée ; l'axe de la voûte correspond au joint $H$ (Fig. 3).

Plan view and vertical cross section across the bottom outlet; the wing wall prevents any water thrust on the thrust block; the joint H (Fig. 3) marks the axis of the arch. 


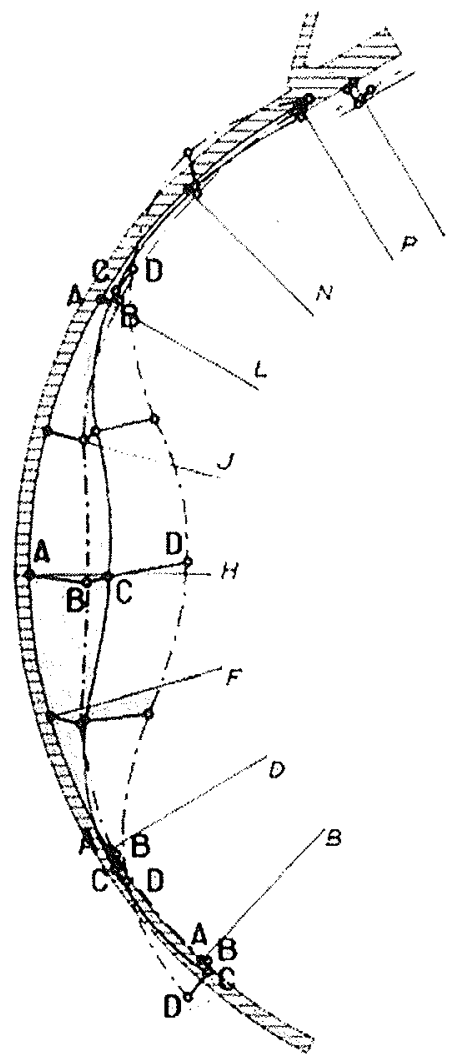

16.3 Plan de l'arc de crête à la cote 98 , portant les lettres $\mathrm{B}$ à $\mathrm{P}$ qui désignent les joints entre les plots de la voûte (un sur deux seulement), et les déformations mesurées aux dates A B C et D (voir Fig. 4) (Mary, 1968, Fig. 23).

Plan-view of the arch at el. 98, showing the letters $B$ to P (names of the joints between monoliths, one of two only) and displacements measured at days A B C D (see Fig. 4) (Mary, 1968, Fig. 23).

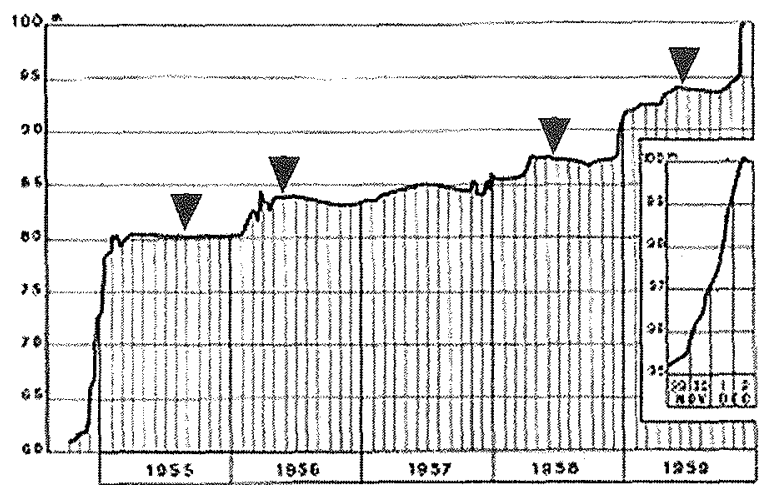

FiG.4 Graphique de remplissage de 1952 à 1959 avec un agrandissement pour les derniers jours : $4,5 \mathrm{~m}$ en 3 jours (soit $1,710^{-5} \mathrm{~m} / \mathrm{s}$ en moyenne) ; les cinq petits crans négatifs signalent des baisses de niveau par ouverture de la vanne de vidange; les quatre triangles situent les opérations d'auscultation topographique (dates A-BC-D de la figure 3 ).

Graph of filling up from 1952 to 1959 , with an enlargement for the last days : $4.5 \mathrm{~m}$ in 3 days (average $1.710^{-5} \mathrm{~m} / \mathrm{s}$ ) ; five little indentations mark openings of the bottom outlet; four triangles point the times of geodesic measurements (dates A-B-C-D on figure 3 ). nelles de l'automne provoquent alors une montée très rapide du 30 novembre au 2 décembre, jusqu'à quelques centimètres sous le seuil déversant. En effet l'exploitant a laissé la vanne fermée afin de protéger le chantier du pont de l'autoroute en activité à moins d'un kilomètre à l'aval (contrairement à la consigne de ne pas dépasser la retenue normale). Après une réunion des autorités concernées sur le site du barrage dans l'après-midi du 2 décembre, la vanne est finalement ouverte à $18 \mathrm{~h}$, mais le niveau n'a que légèrement baissé lorsque le barrage disparaît à $21 \mathrm{~h} 10$.

\section{6}

\section{Premières constatations}

L'examen des lieux (Figs. 5 à $9^{4}$ ) montre qu'une partie du barrage subsiste en rive droite et en fond de vallée, presque jusqu'au joint K. Au pied aval, le bassin a disparu, laissant place à une large fosse à la cote 35 (d'après les coupes dans Mary, 1968), et en rive gauche un grand volume de terrain de fondation a été emporté avec l'ensemble des plots de béton $\left(40000 \mathrm{~m}^{3}\right.$

(4) Photos de Joseph Duffaut (J.D.), envoyé sur place par le ministre des Travaux publics dès le 3 décembre en qualité de chef du ser vice technicque de l'électricité et des grands barrages, cui a fait partie ensuite de la Commission d'enquête administrative dont la première visite a eu lieu le 20 décembre et de Pierre Duffaut (P.D.) cui a visité le site au printemps 1960, avant d'hériter des photos paternelles, et d'en prendre lui-même lors des nombreuses visites effectuées depuis.

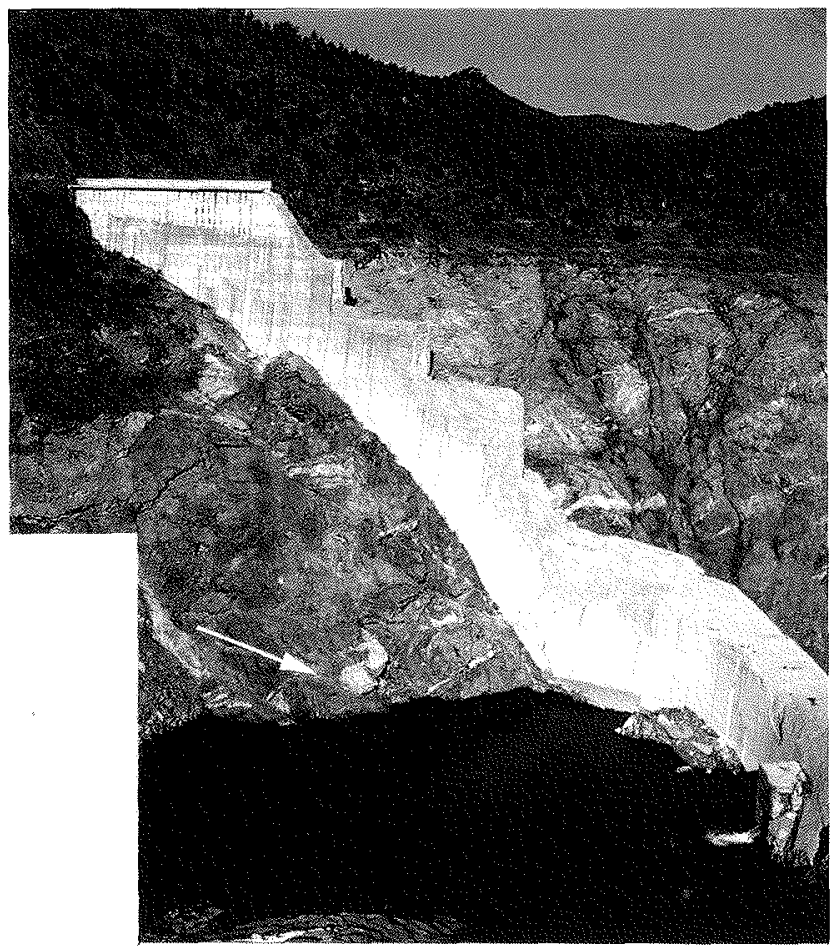

\$6. 5 La rive droite du barrage est découpée en escaliers suivant les joints de construction et les reprises de bétonnage ; la photo, P.D. du printemps 1960 , montre le rocher nettoyé par le déplacement de l'eau ; la flèche indique la même faille que sur la figure 6.

The right bank remains of the dam are cut in stairs along construction joints and levels of pouring concrete; photo by P.D. in spring 1960 showing the rock cleaned up by the flow of water : the arrow shows the same fault as on figure 6 . 


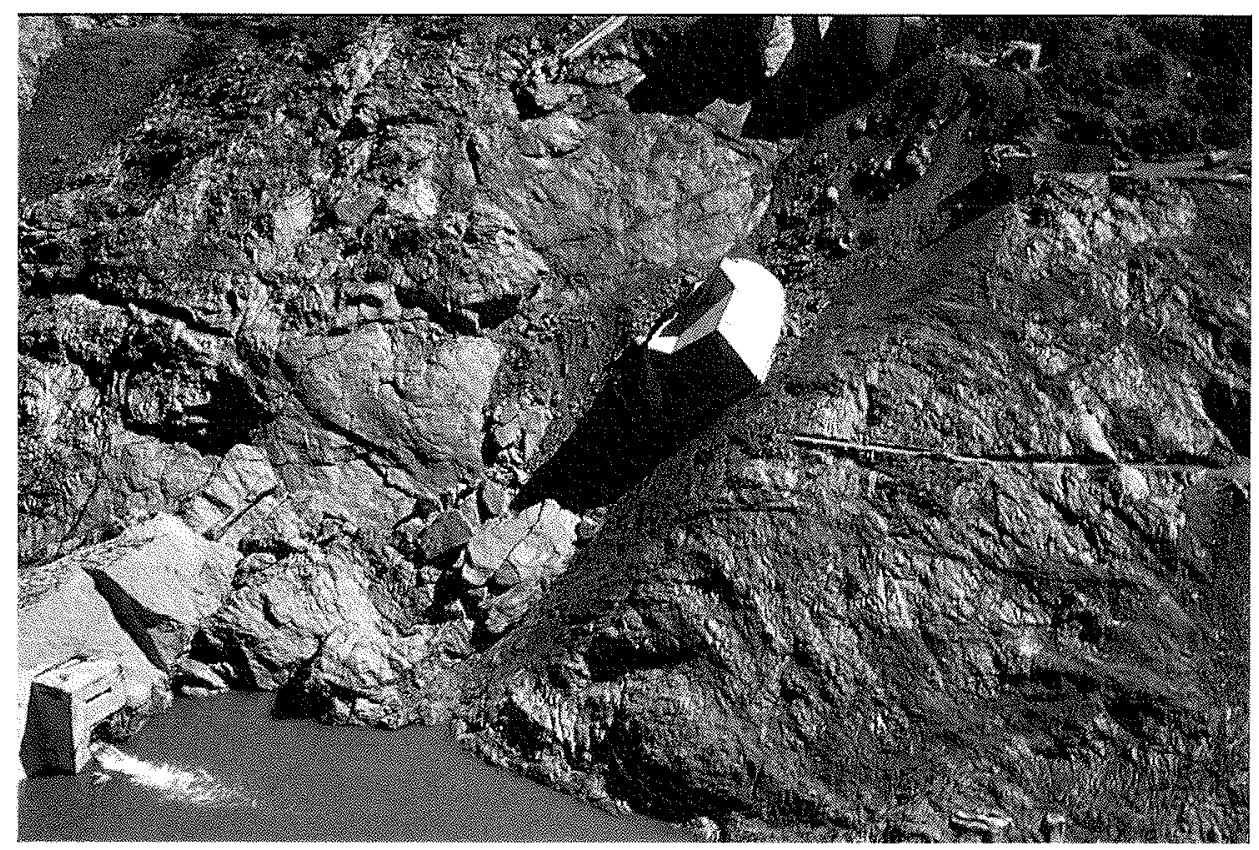

F6. 6 Trait caractéristique de la rive gauche, le dièdre excavé au pied du barrage et le bloc de culée qui y est tombé ; à l'extrême droite s'ouvre la galerie creusée pour les essais au vérin (on voit aussi de part et d'autre la trace de l'aqueduc romain de Fréjus, à la cote 53) (photo P.D., printemps 1960).

Characteristic feature of the left bank, the dihedron excavated at the dam foot and the concrete block fallen inside from the thrust block; at right opens the adit for jack tests (remains of the roman aqueduct keep visible upstream and downstream at el. 53) (photo P.D., spring 1960).

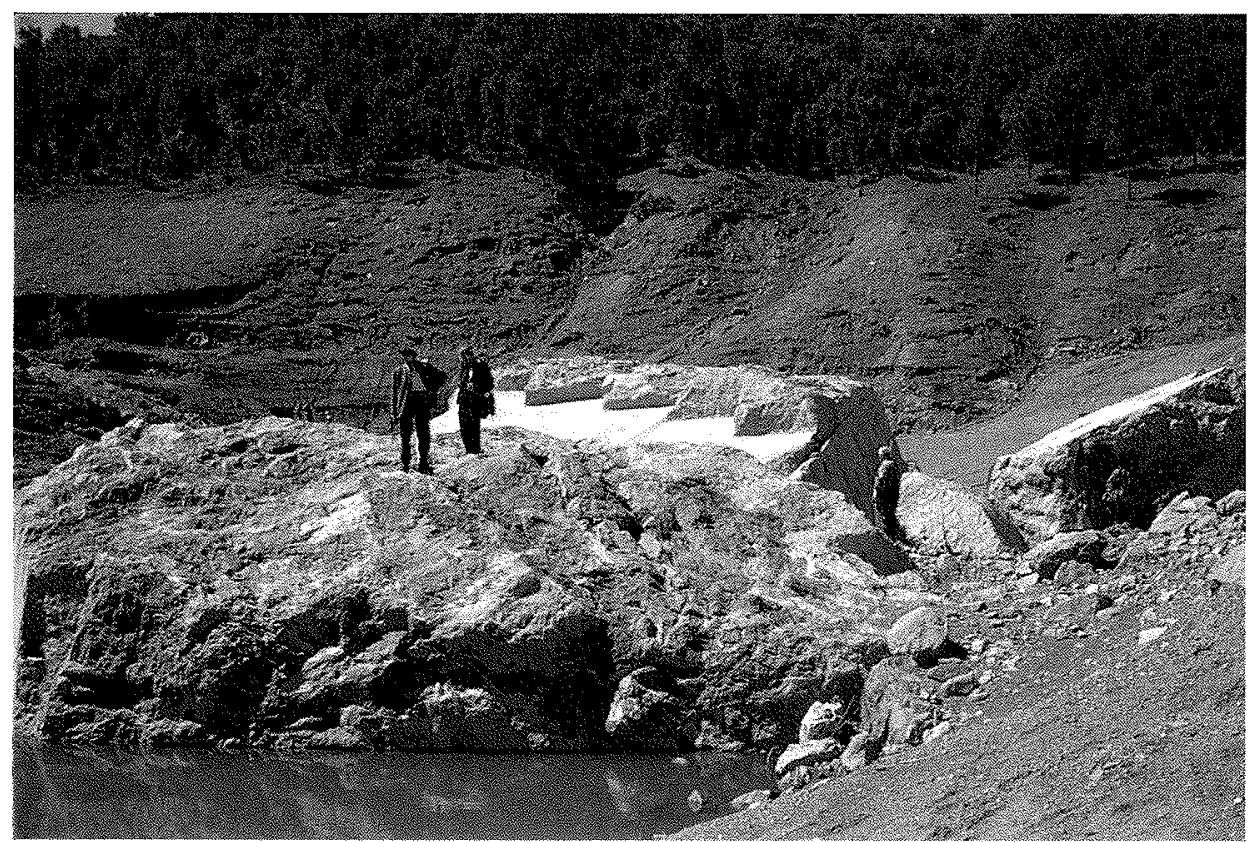

f6. 7 Les deux principaux blocs de béton, transportés à plus de $500 \mathrm{~m}$, montrant le rocher attenant au béton (photo J.D. du 20/12/59).

Two main concrete blocks, carried away more than $500 \mathrm{~m}$, showing the rock sticking on their basal concrete surface (photo J.D. of 20/12/59)

environ). Ce volume manquant (Fig. 6) est décrit schématiquement comme un dièdre, dont la face aval est un plan de faille caractérisé, lisse et strié, la face amont un ensemble de déchirures suivant des surfaces de foliation irrégulières (plutôt que des failles comme il a pu être écrit). Le premier plot PQ de la culée est tombé au creux de ce dièdre en restant solidaire d'une petite partie du mur en aile.
La surface de la faille ainsi mise en évidence a une direction perpendiculaire à la vallée ; on la retrouve au pied de la rive droite (Figs. 5 et 8); son pendage de $40^{\circ}$ environ la fait passer à $20 \mathrm{~m}$ de profondeur sous le barrage (comme il apparaît sur les figures 10 et 15 ).

La vallée à l'aval est parsemée d'énormes blocs de béton, accumulés à l'amont de chaque coude, dont les principaux ont pu être identifiés. Les bases de plots 


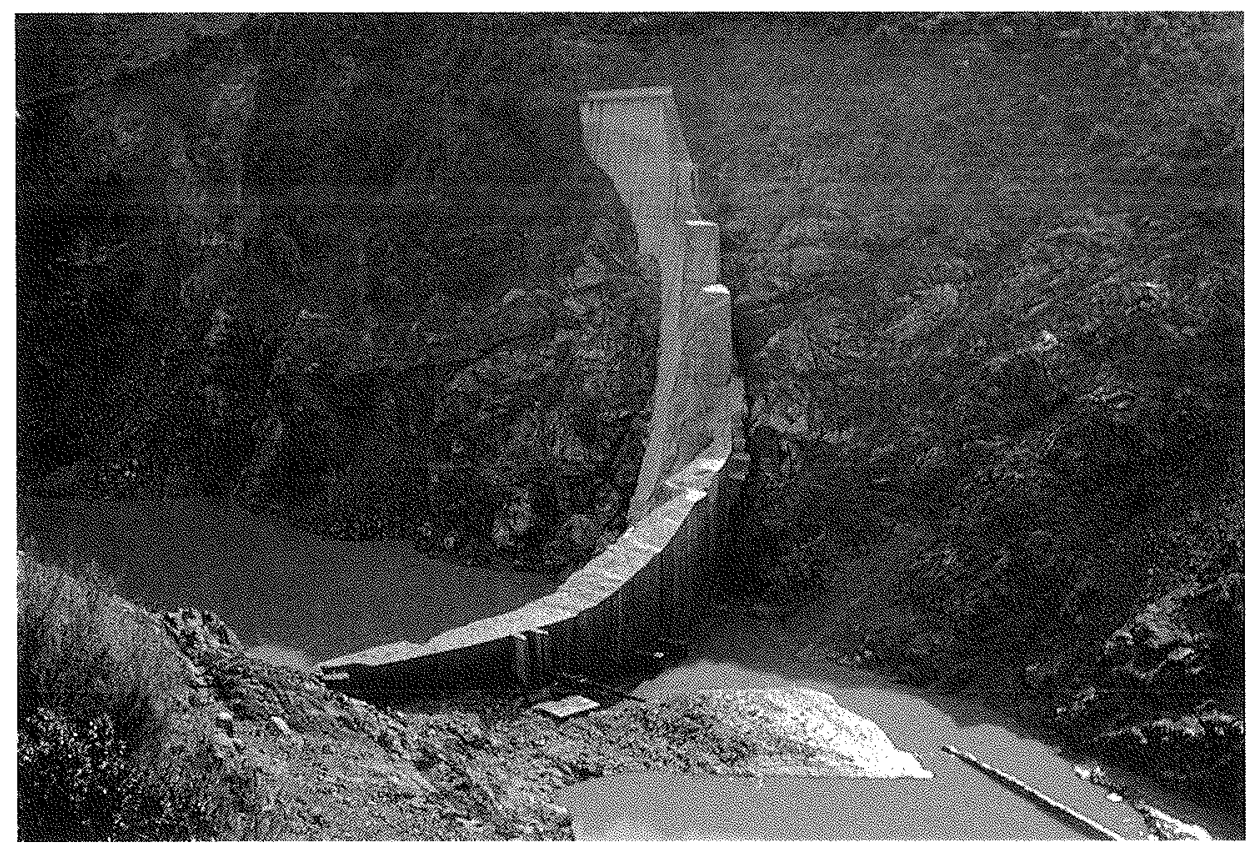

16. 8 Trait caractéristique de la rive droite, la crevasse large et continue qui suit le pied amont du béton (photo J.D. du 20/12/59) ; les reprises de bétonnage luisent au soleil ; le mur de soutènement de l'ancienne plateforme ferroviaire apparaît à droite.

Characteristic feature of the right bank, the wide crevice following the heel of the clam (photo J.D. of 20/12/59); concrete levels shine in the sun; the retaining wall of the old railroad platform appears at right.

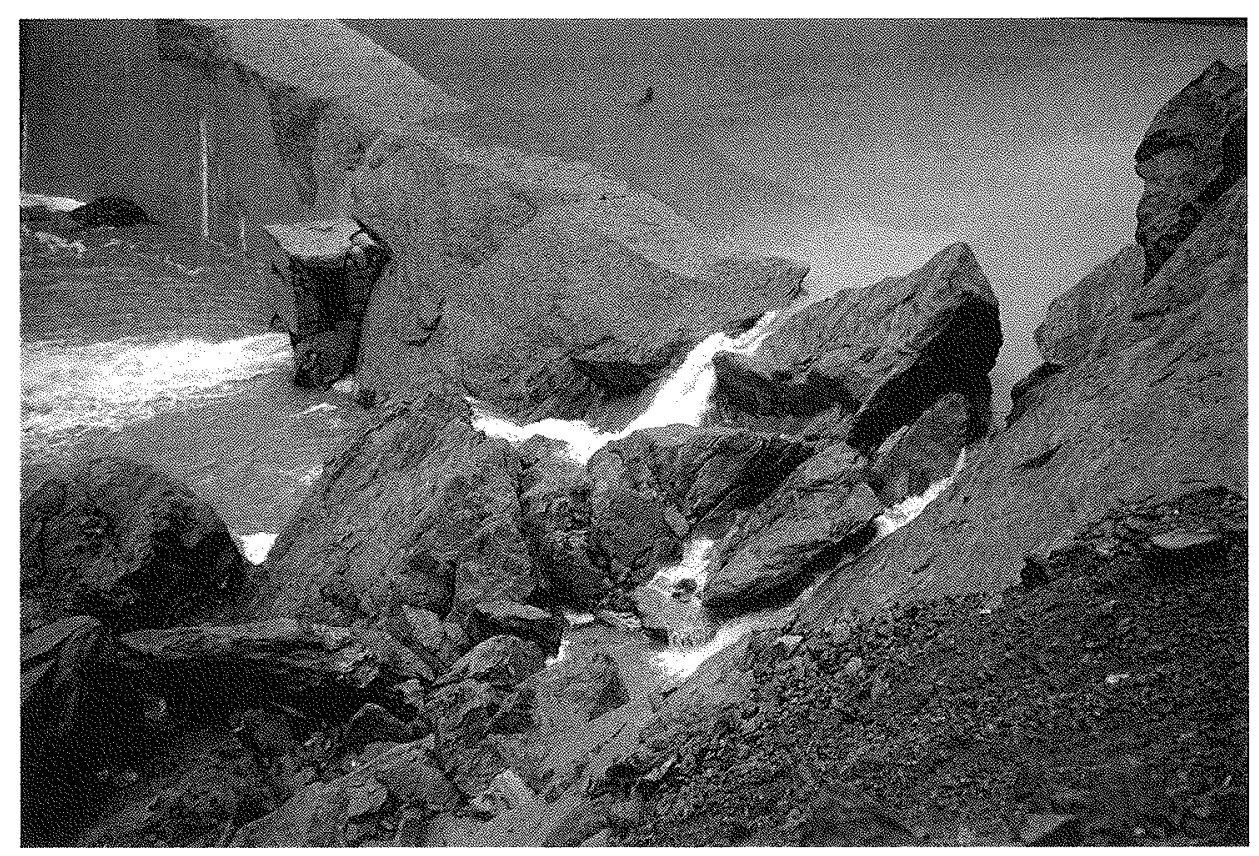

Fic. . Débordement par-dessus le béton au pied de la rive gauche (photo J.D. du 4/12/59); l'écume visible en haut à gauche signale l'eau qui passe sous le plot $\mathrm{FG}$.

Overflow over the concrete at the foot of left bank (photo J.D. of 4/12/59); foam visible at left marks water crossing under monolith FG.

montrent une certaine épaisseur de rocher attenant au béton, attestant que la rupture s'est produite au sein du rocher (Fig. 7).

Sur la rive droite (Fig. 8), une crevasse large et profonde apparaît au pied amont des plots qui subsistent, témoignant d'un déplacement de la voûte vers l'aval, que le terrain situé à l'amont n'a pas suivi. Sur les deux rives le rocher a été déshabillé de toute végétation et terre végétale par le passage du flot ${ }^{5}$; des éboulis postérieurs à la rupture frangent le pied cle la rive droite (Fig. 5) et le haut du dièdre. Le béton qui subsiste en rive droite est découpé en marches d'escalier par les joints de construction $C, D$ et $E$, par des surfaces de

(5) « La catastrophe ayant enlevé tous les terrains superficiels, les conditions d'observation étaient infiniment supérieures aux conditions antérieures » (déposition de Jean Goguel au procès). 
reprise de bétonnage sur toute la surface des plots IJ et JK et une partie des plots de BC à GH, et localement par des surfaces moins régulières.

Le béton au pied de la rive est parcouru de fractures nombreuses évoquant une torsion de la coque face au bajoyer rive droite du bassin de réception des crues (Fig. 11). Les arêtes aval des plots CD et DE sont fortement épaufrées. Les surfaces de rupture du béton montrent partout une excellente qualité apparente (que confirmeront les essais effectués au Laboratoire central des ponts et chaussées sur des carottes prélevée par forage).

Les 3 et 4 décembre, l'eau déborde encore par-dessus la limite du béton au pied de la rive gauche et sur le plot IJ (Fig. 9), puis le niveau amont baisse lentement, l'eau s'écoulant à la fois par la vidange de fond et pardessous le barrage, notamment sous le plot FG, puis uniquement par-dessous le béton (des nappes d'eau subsisteront des deux côtés jusqu'à la sécheresse de 1962, empêchant l'examen des parties inférieures du barrage).

\section{4}

\section{Étapes des études engagées après la rupture}

Une commission d'enquête administrative est nommée le 5 décembre par le gouvernement, et deux commissions d'expertise seront nommées successivement par le tribunal de Draguignan, le 12 décembre 1959, puis le 7 mai 1962 (Tableau II). La commission administrative s'adjoindra aussitôt le géologue Jean Goguel, alors vice-président du Bureau de recherches géologiques et minières, qui procédera dès le 20 décembre à un examen approfondi du terrain.

Ces commissions font procéder d'abord à un lever topographique précis, ensuite à tous les essais disponibles, sur le terrain et en laboratoire, et elles font reprendre tous les calculs. De nombreux échantillons de roche sont prélevés et confiés à divers laboratoires (dont celui de l'École polytechnique), un petit puits et une galerie sont creusés rive gauche pour accueillir les essais de déformabilité. De leurs côtés, le bureau $\mathrm{ACJB}^{6}$ et son président André Coyne (qui avait fourni le projet de barrage), ainsi que EDF (intéressé au plus haut degré car ayant la responsabilité de très nombreux barrages, dont beaucoup de barrages-voûtes) vont mettre en route un certain nombre de calculs et d'essais en sus de ceux demandés par les commissions.

(6) Le bureau d'études fondé par André Coyne a d'abord été nommé ACJB (André Coyne et Jean Bellier) puis est devenu plus tard COB (Coyne et Bellier).
Le lever topographique met immédiatement en évidence la rotation d'ensemble de ce qui reste du barrage, à partir d'un point fixe à l'extrémité rive droite : les déplacements augmentent en effet régulièrement jusqu'à $80 \mathrm{~cm}$ pour la portion qui subsiste, la culée ayant avancé de plus de $2 \mathrm{~m}$, deux fois plus que ce qu'implique la rotation ci-dessus. Les mesures sismiques qui ont quadrillé le versant rive gauche montrent que la célérité longitudinale du terrain, excellente en profondeur, est fortement diminuée dans une épaisseur de dix à vingt mètres à partir de la surface.

EDF envoie sur place dès le printemps une équipe spécialisée dans les mesures de déformabilité des massifs rocheux, et aussitôt après engage l'exécution de mesures semblables sur tous les chantiers de barrages en cours aux fins de comparaison.

Désignés en raison de désaccords persistants entre les experts de la commission administrative et ceux de la première commission judiciaire, les contre-experts engagent des reconnaissances complémentaires, facilitées par la sécheresse de 1962 qui a permis pour la première fois l'accès au pied de la voûte et même sous le barrage : la réalité d'un passage sous le plot FG par une crevasse ouverte au sein du rocher est ainsi démontrée (Fig. 10) ; la disposition des dépôts de part et d'autre laisse penser qu'un débit important a transité sous ce plot après l'essentiel du déversement, comme le suggère le seuil en arc de cercle visible sur la figure 11.

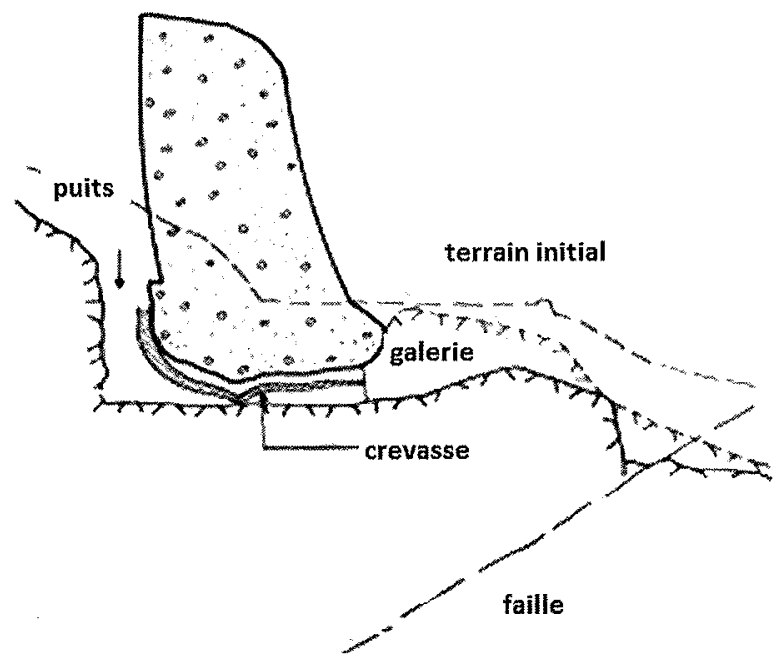

16. 10 Coupe sous le plot FG, où la crevasse dans le rocher de fondation a été mise en évidence le long d'une petite galerie creusée en 1962.

Cross-section under monolith FG, where the crevice through the foundation rock has been made obvious along a small gallery bored 1962.

TABLAu II Composition des trois commissions d'enquête sur la rupture de Malpasset (IG, ingénieur ou inspecteur général ; EDF, Électricité de France ; TP, travaux publics ; CFGB, Comité français des grands barrages ; GTM, Grands travaux de Marseille; PC, Ponts et Chaussées).

Experts nonnmes part le ministre

Gosselin, IG Ponts et Chaussées

Olivier-Martin, directeur à EDF

Duffaut J., IG, ministère TP

Talureau, IG Génie rural

Calvet IG Génie rural

Disserens, ing. conseil, GTM

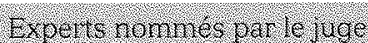

Casteras, prof. géologie

Escande, dir. école d'Hydraulique Gridel, prof. Centrale

Haegelen, IG Ponts et Chaussées

Jacobson, prof. École centrale

Roubault, dir. école de Géologie

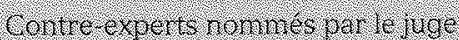

Caquot, membre de l'Institut Pruvost, membre de l'Institut Mary, PC, ancien pt CFGB

Barbier, prof. géologie

Biarez, prof. École centrale

Drouhin, PC, président CFGB 


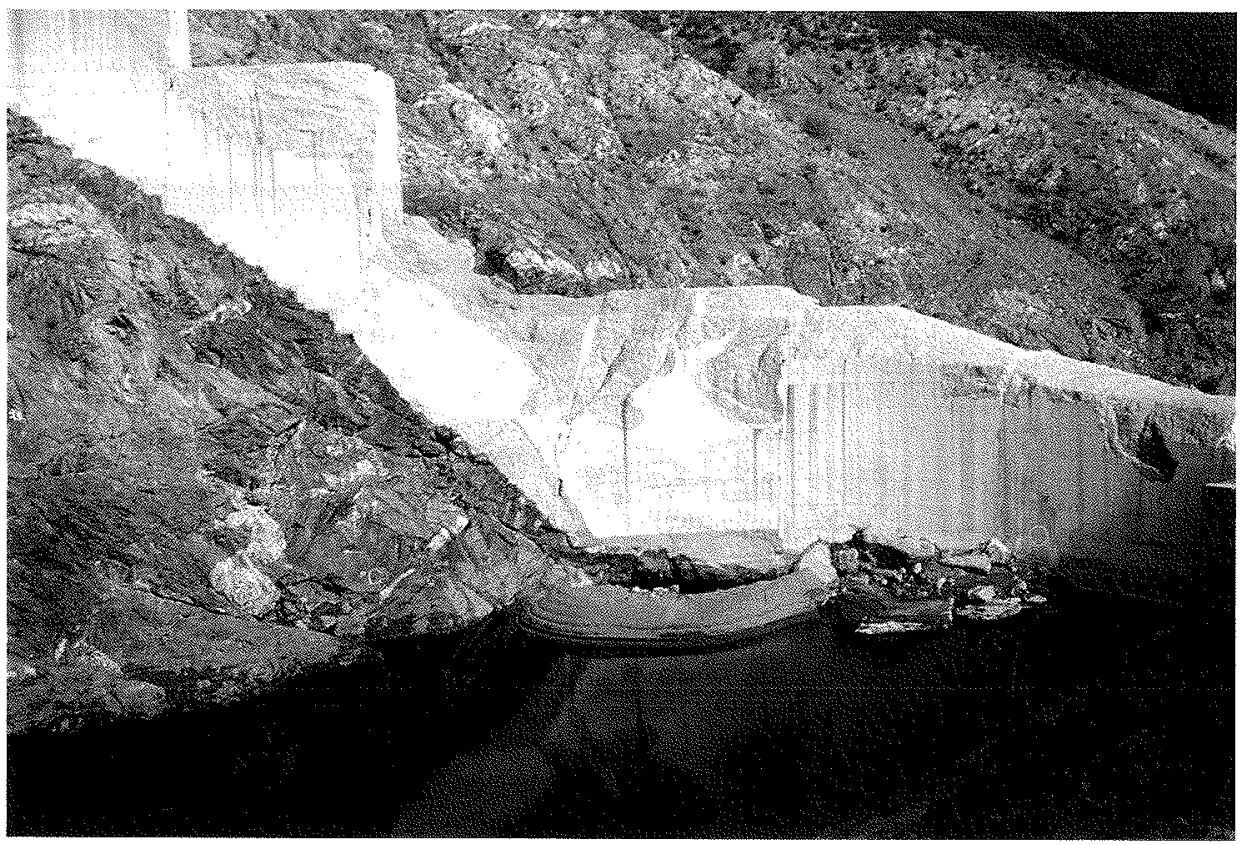

FG 11 닐 Photo P.D., printemps 1960, montrant le seuil construit par le passage de l'eau dans la crevasse sous le plot FG.

Photo P.D., spring 1960, showing the sill built by the water flow through the crevice under monolith FG.

\section{3}

\section{Études de mécanique des roches sur le terrain}

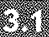

\section{La déformabilité du terrain in situ}

La division génie civil du service Études et projets hydrauliques d'EDF (direction de l'équipement) a standardisé (Talobre, 1957) une méthode d'essai sur le terrain, à partir de l'essai de chargement « à la plaque » utilisé en mécanique des sols ; l'échelle des efforts nécessaires fait appel au chargement par un vérin hydraulique d'une plaque circulaire rigide (diamètre $28 \mathrm{~cm}$, surface $615 \mathrm{~cm}^{2}$ ) avec la réaction fournie par la paroi opposée d'une excavation (galerie ou puits), d'où la dénomination classique d'essais « au vérin » (jack tests en anglais). La contrainte appliquée était alors le plus souvent limitée à 70 ou $80 \mathrm{~kg} / \mathrm{cm}^{2}$ (7 ou $8 \mathrm{MPa}$ ) mais elle a atteint ailleurs 4 fois plus. EDF entretient à l'époque une équipe qui dispose du matériel néces saire et l'emploie régulièrement pour l'étude du terrain autour des conduites forcées souterraines. Pour le barrage de Tignes, fondé sur des quartzites très durs, EDF avait fait appel au CEBTP pour de tels essais (Habib. 1950), alors que les usages précédents en matière de fondations de barrages n'avaient porté que sur des roches nettement plus tendres en Algérie (Drouhin, 1936) et en Suisse, ou sur des ouvrages exceptionnels (Hoover dam, États-Unis, Grande Dixence, Suisse).

Deux galeries de petit diamètre ont été creusées spécialement dans le versant rive gauche (repères 1 et 2 sur figure 12), à la cote 53 ; la première, longue de $35 \mathrm{~m}$, a accueilli 5 postes de mesure, la seconde, de $8 \mathrm{~m}$ seulement, un seul poste; l'emplacement des postes a été choisi après une étude microsismique le long de la première galerie; 19 essais ont été exécutés, dont 11 en position verticale ( 6 en calotte et 5 en radier) et 8 en position horizontale.

Le graphique de la figure 13 rassemble les résultats obtenus à Malpasset et sur 16 autres sites de barrages (Tableau III) ; une première publication, au congrès des grands barrages d'Edimbourg en 1964 (Groupe de travail ${ }^{7}$ ), en comportait 8 , alors en construction ou à l'étude en France pour EDF. EDF en a ajouté plus tard 8 autres (graphique cité au colloque de Purdue, Post et Bonazzi, 1987).

La comparaison est sans appel : Malpasset occupe de loin la dernière place; et on peut noter que le site de Saint-Cassien, qui est très proche au nord-est de Malpasset, dans le même massif cristallin ancien, a des valeurs nettement supérieures (médiane multipliée par 3) ; c'est l'épaisseur de la zone d'altération superficielle qui a conduit à éliminer toute solution en béton sur ce site. Les projets initiaux de Saint-Cassien et de Roujanel étaient des voûtes : EDF a maintenu celui-ci, qui se classait pourtant avant-dernier des voûtes sur le Tableau, au prix du traitement d'une faille qui était bien visible sur le terrain (contrairement à celle de Malpasset) mais a préféré la solution enrochement pour SaintCassien comme pour Besserve.

Parmi les sites ajoutés plus tard, plusieurs concernent des barrages de plus grande hauteur (Tignes, Monteynard, Vouglans, Sainte-Croix). Entre Malpasset et Laparan, le graphique montre que les quinze autres se rangent en deux groupes intermédiaires, l'un avec une médiane de 6 à $10000 \mathrm{MPa}$ (Puylaurent et Laouzas sur du granite, Tignes sur des quartzites, Monteynard, Vouglans et Sainte-Croix sur des calcaires), l'autre dont la médiane est entre 1000 et $2500 \mathrm{MPa}$. On note que

\footnotetext{
(7) Pierre Habib était co-président de ce groupe de travail et Pierre
} Duffaut assurait le secrétariat des quatre groupes. 


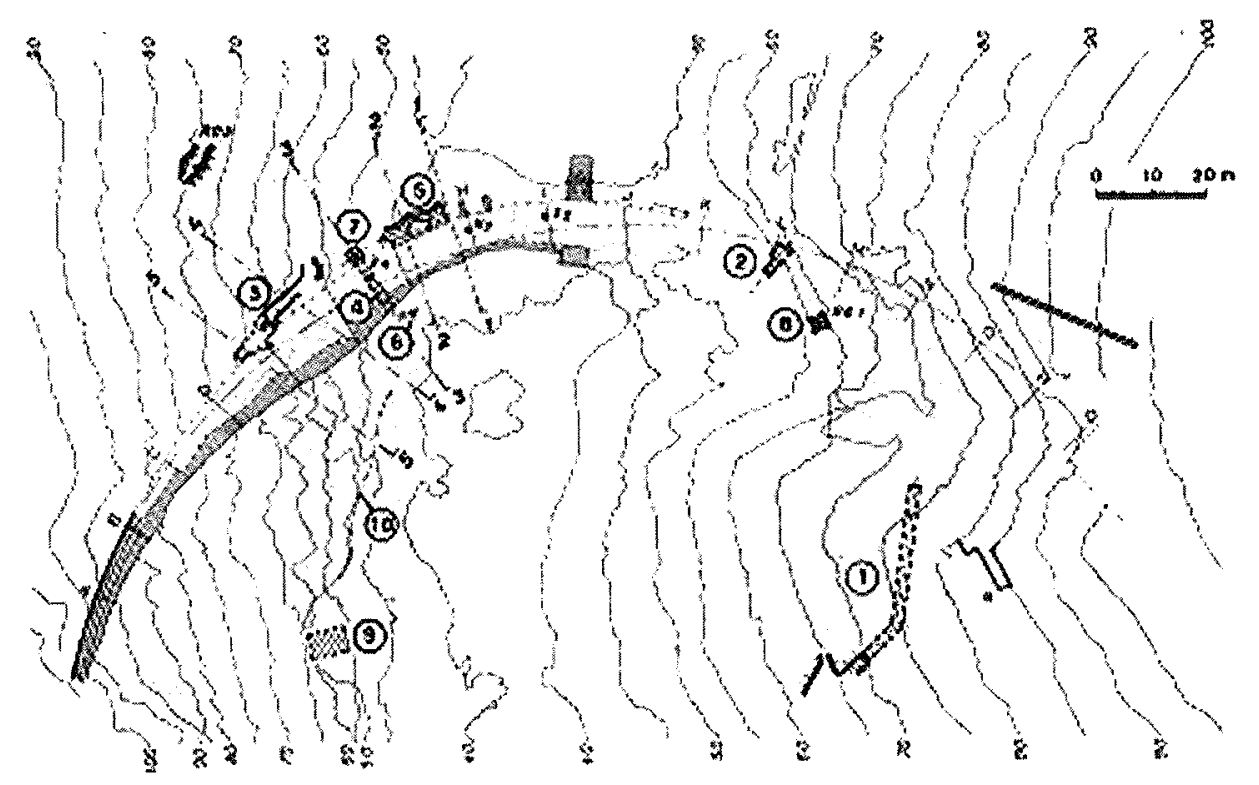

Fc. 12. Implantation des galeries et puits de reconnaissance. Position of exploratory adits and pits.

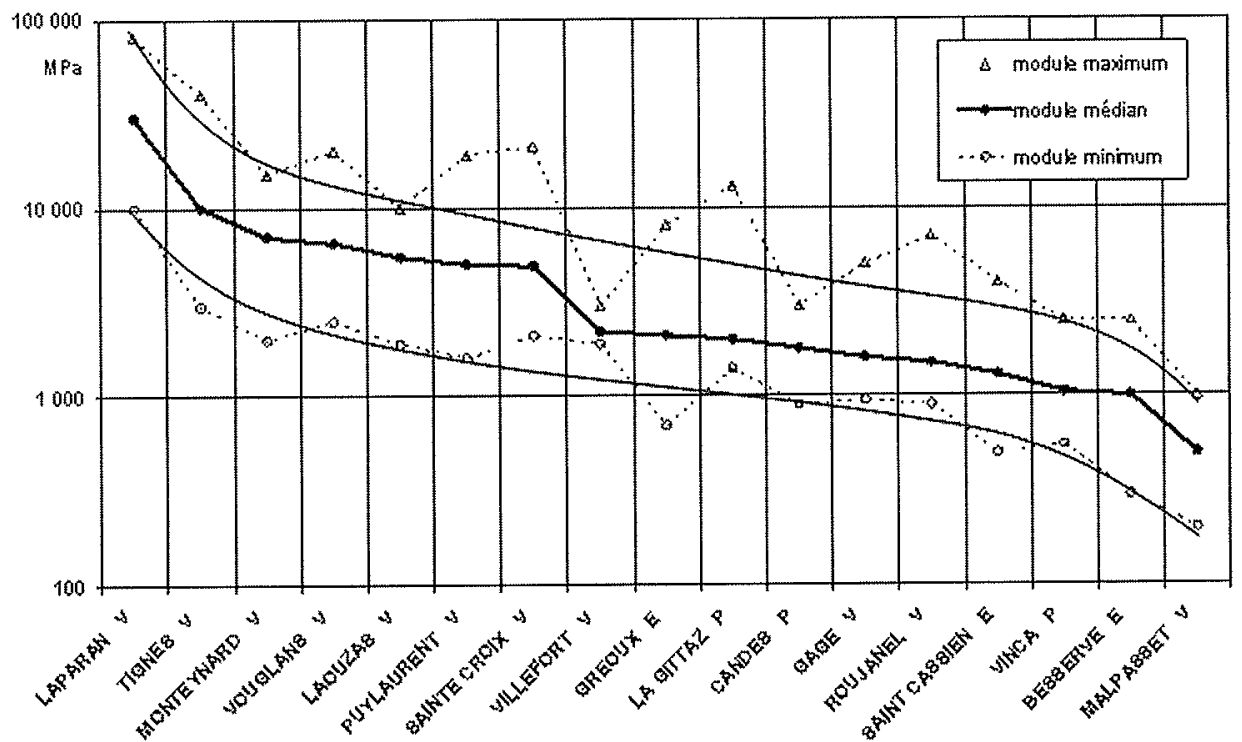

16. 13. Graphique des modules " de déformation ) du terrain mesurés sur 17 sites de barrages ( 11 voûtes, 3 poids, 3 enrochements) ; l'échelle des modules, en MPa est logarithmique. Les sites sont rangés par modules médians décroissants (courbe centrale, entre valeurs extrêmes) (dessin de $B$. Goguel d'après Post et Bonazzi in Leonards, 1987).

Graph of deformation modules of the ground measured on 17 dam sites (11 arch dams, 3 gravity dams, 3 rock-fill dams); the MPa scale is logarithmic. Dam sites are sorted along decreasing median modules (bold curve, in between curves of extreme values) (drawing by B. Goguel after Post and Bonazzi in Leonards, 1987).

les voûtes constituent plus de la moitié de l'effectif, dont les huit sites les mieux classés et deux dans le groupe inférieur (sans compter Malpasset qui clôt la liste, comme dans le premier groupe étudié). On sait que le module lu sur les cycles de déformation est plus élevé que celui qui apparaît au premier chargement, au moins deux fois supérieur en général, ce qui était confirmé à Malpasset.

\section{3.}

\section{Conséquences d'une grande déformabilité}

Pourquoi ce module est-il aussi critique? A l'époque du projet, la mesure de la déformabilité des fondations de barrages était rare, réservée à des cas extrêmes de terrains jugés relativement mous, ou au contraire très rigides; il était d'usage de (calculer») les voûtes en 


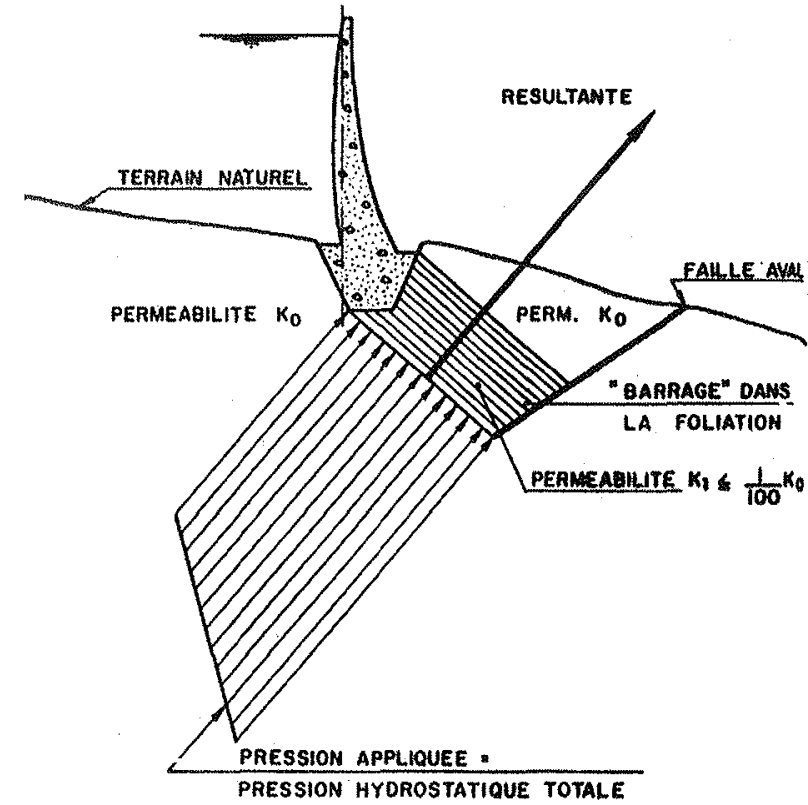

16. 14 Schéma du " barrage » supplémentaire provoqué au sein du terrain par la diminution de sa perméabilité sous l'effet de la poussée du barrage : la résultante de la pression dans la foliation est susceptible de chasser le dièdre le long de la faille (d'après Mary, 1968).

Diagram showing that the hydrostatic pressure insicle the foliation against the extra " dam $x$ built inside the ground by the decrease of its permeability induced by the dam thrust may push the dihedron along the fault (after Mary 1968).

prenant pour le rocher un module de l'ordre de la moitié de celui du béton. On s'accommodait de modules diminuant du pied des versants vers le haut, puisque la rigidité des barrages diminuait aussi. Après la rupture du rocher de Malpasset, les ingénieurs se sont partagés en deux groupes suivant l'importance attribuée à la déformabilité du terrain : ce rocher était-il trop mou, comme quelques voix le dénonçaient? Alors qu'une majorité penchait pour ne trouver aucun inconvénient à cette grande déformabilité. Mary (1968) écrit que la valeur déduite « du déplacement radial du pied de l'ouvrage sous l'action de l'effort tranchant des consoles se situerait aux environs de 10000 bars (1 000 MPa) et peut-être moins » et conclut que « ce module est faible ». Cette valeur, déduite d'un calcul effectué par COB, est rappelée à Purdue par Post et Bonazzi (1987). D'après Bellier (1967) on avait pris un rapport dix pour le calcul de la voûte de Malpasset, et la réalité était probablement au-delà.

Cette déformabilité a d'ailleurs une conséquence qui ne met pas en cause la façon de travailler de la structure du barrage : sous la poussée de l'eau, tout barrage avance en comprimant sa fondation à l'aval, et avance d'autant plus (à hauteur d'eau égale) que le module du rocher est plus faible. Faute de résister à un allongement, le massif rocheux situé à l'amont immédiat ne suit pas le barrage, ce qui se traduit par l'ouverture d'une ou plusieurs fissures. Ce phénomène, qui passe inaperçu lorsque la fondation est rigide même pour de hauts barrages (des ouvertures millimétriques ont été mesurées à Vouglans grâce à une auscultation spécialement mise au point) et aussi sous les barrages poids, toujours épais, en béton ou davantage encore en remblais, prend de l'importance à Malpasset, où l'avancée du barrage a dépassé le centimètre en clé sous une charge encore partielle, et il peut expliquer l'ouverture finale de plusieurs décimètres de la crevasse découverte au pied amont des plots de rive droite, après la rotation en bloc du barrage. Comme la déformabilité était plus grande en rive gauche, une crevasse plus large encore a dû s'y ouvrir (favorisée par la direction des surfaces de foliation, et en accord avec la rotation d'ensemble)

\begin{tabular}{|c|c|c|c|c|c|c|c|}
\hline \multirow{2}{*}{$\begin{array}{l}\text { TABLEU in } \\
\text { noll }\end{array}$} & \multicolumn{7}{|c|}{$\begin{array}{l}\text { Données principales des barrages français de la figure 14, dans le même ordre. } \\
\text { Main data on French dams of figure 14, in the same order. }\end{array}$} \\
\hline & rinee & Prorere & Departernent & Terrant & rue & Tarcelo & Exploilstil \\
\hline Laparan & 1985 & Aston & Ariège & granite & voûte & 106 & EDF \\
\hline Tignes & 1952 & Isère & Savoie & quartzite & voûte & 180 & EDF \\
\hline Monteynard & 1963 & Drac & Isère & calcaire & voûte & 155 & EDF \\
\hline Vouglans & 1968 & Ain & Ain & calcaire & voûte & 130 & EDF \\
\hline Laouzas & 1965 & Vèbre & Tarn & granite & voûte & 52 & EDF \\
\hline Puylaurent & 1996 & Chassezac & Lozère & granite & voûte & 73 & EDF \\
\hline Sainte-Croix & 1974 & Verdon & Alpes-H.-Prov. & calcaire & voûte & 95 & EDF \\
\hline Villefort & 1965 & Altier & Lozère & granite & voûte & 75 & EDF \\
\hline Gréoux & 1967 & Verdon & Alpes-H.-Prov. & calcaire & enrochements & 67 & EDF \\
\hline Gittaz (la) & 1967 & Gittaz & Savoie & gneiss & poids & 67 & EDF \\
\hline Candes & 1967 & R. de Candes & Lot & granite & poids & 54 & EDF \\
\hline Gage II (le) & 1967 & Gage & Ardèche & granite & voûte & 42 & EDF \\
\hline Roujanel (le) & 1965 & Borne & Ardèche & gneiss & voûte & 57 & EDF \\
\hline Saint-Cassien & 1966 & Biançon & Alpes-Mmes & gneiss & enrochements & 66 & EDF \\
\hline Vinça & 1977 & Têt & Pyrénées-Or: & granite & poids & 60 & Pyr.-Or. \\
\hline Besserve & 1968 & Sioule & Allier & gneiss & enrochements & 68 & EDF \\
\hline Malpasset & 1954 & Reyran & Var & gneiss & voûte & 60 & Var \\
\hline
\end{tabular}


sans même nécessiter aucun début de glissement sur le plan de la faille formant la face aval du dièdre. Plus la crevasse amont s'ouvre en surface, plus elle s'étend en profondeur, donnant à l'eau de la retenue une action plus profonde comme si la hauteur du barrage avait augmenté (on sait que la force augmente alors comme le carré de la hauteur) (Fig. 15, Mary, 1968).

\section{Composition des forces sur le terrain de fondation d'un barrage}

\section{4.}

\section{Le schéma des contre-experts}

C'est l'essentiel du schéma retenu par les contreexperts et résumé par Mary (1968, p. 77) en deux phases, suivant la figure 15 de Laginha Serafim (in Leonards, 1987, p. 356)

Première phase, qui a probablement débuté longtemps avant la rupture sans attirer l’attention : « Sous l'action des poussées exercées par le barrage et des pressions interstitielles, le pied s'est légèrement déplacé, ... permettant à l'eau de pénétrer progressivement dans la crevasse...; lorsque la pression de l'eau du lac a pu s'exercer dans toute l'étendue de cette crevasse et avec sa pleine valeur du fait de l'imperméabilisation de toute la zone intéressée par le jeu des forces de compression exercées par le barrage, le rocher situé à l'aval a été expulsé brutalement... ».

Conséquence (deuxième phase) : “ la poussée de la voûte, privée de son appui sur une grande longueur, s'est concentrée sur la culée qui a dès lors été soumise à une force excessive, ... L'élément résistant clu barrage était alors constitué par un arc oblique... prenant appui sur la culée... Cet arc, chargé d'une manière excessive a péri, peut-être par flambement ». En fait la dérobade de la culée a annulé l'effet d'arc, supprimant tout risque de flambement, et les consoles ont péri surtout par flexion, comme il est écrit sur la figure 15.

\section{3}

\section{Généralisation}

Pierre Londe (1965) va généraliser ce type de schéma à trois dimensions pour pouvoir l'adapter à tout bloc individualisable au sein d'une rive rocheuse (les faces du bloc rocheux étant des joints, au sens anglais, c'està-dire aussi bien joints de stratification ou schistosité, que des fractures ou failles) : l'abaque qu'il publie dès 1965 va beaucoup plus loin car il permet de discuter la stabilité d'un bloc rocheux tétraédrique en fonction des forces exercées sur ses faces (poids propre, poussées, pressions et sous-pressions) et des coefficients de frottement (Figs. 16 et 17). Il permet donc d'estimer la nécessité d'un drainage localisé et d'en apprécier l'efficacité (analyse détaillée dans Carrère 2010).

\section{5}

\section{Conclusion:}

\section{vers une nouvelle mécanique des roches}

\section{0.}

\section{Les suites judiciaires}

Le concepteur, André Coyne, avait aussitôt revendiqué sa responsabilité : “ je suis le seul coupable », à tort évidemment lorsqu'on considère les années d'exploitation sans surveillance pendant lesquelles le Bureau ACJB n'avait aucun contrat de suivi; beaucoup de barrages sont modifiés au cours de leurs premières années (Tolla en Corse, Roselend en Savoie, Kölnbrein en Autriche, par exemple), et quelques-uns sont

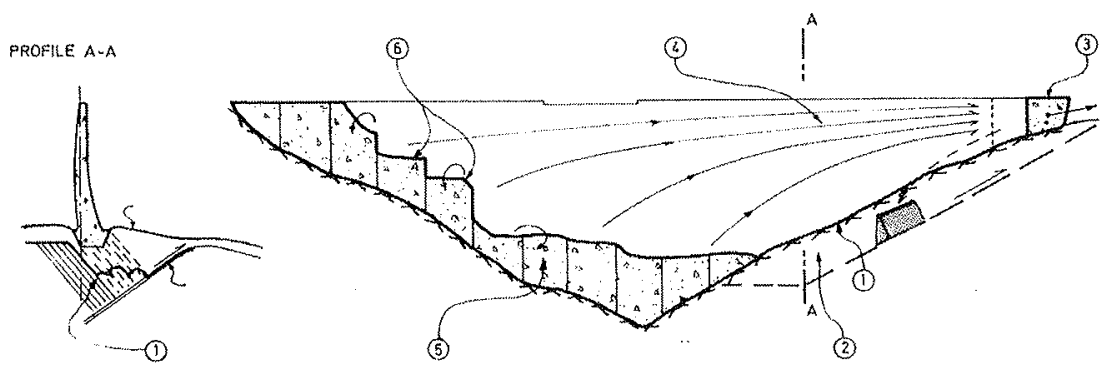

nc. 15 A gauche coupe à mi-hauteur de la rive gauche : la poussée de l'eau étanche le terrain et ouvre la foliation, l'eau pénètre la crevasse (1) ; à droite le dièdre ayant commencé à glisser vers l'aval et vers le haut (2), la coque reporte sa poussée vers la culée (3), qui cède (4); l'arc inférieur fléchit horizontalement (5); privée d'appui en rive gauche, les plots rive droite fléchissent verticalement (6) et la coque explose (d'après Serafim, in Leonards, 1987).

Left, cross section of the dam at mid height of left bank : the thrust on the dam tightens the ground and opens it along foliation planes; water enters the crevice (1) ; right when the dihedron has begun to slide towards downstream and upwards (2), the shell transfers its thrust onto the thrust block (3), which gives up (4); the lower arches bend horizontally $(5)$; the monoliths on the right bank bend vertically $(6)$ and the whole shell bursts (after Serafim, in Leonards, 1987). 

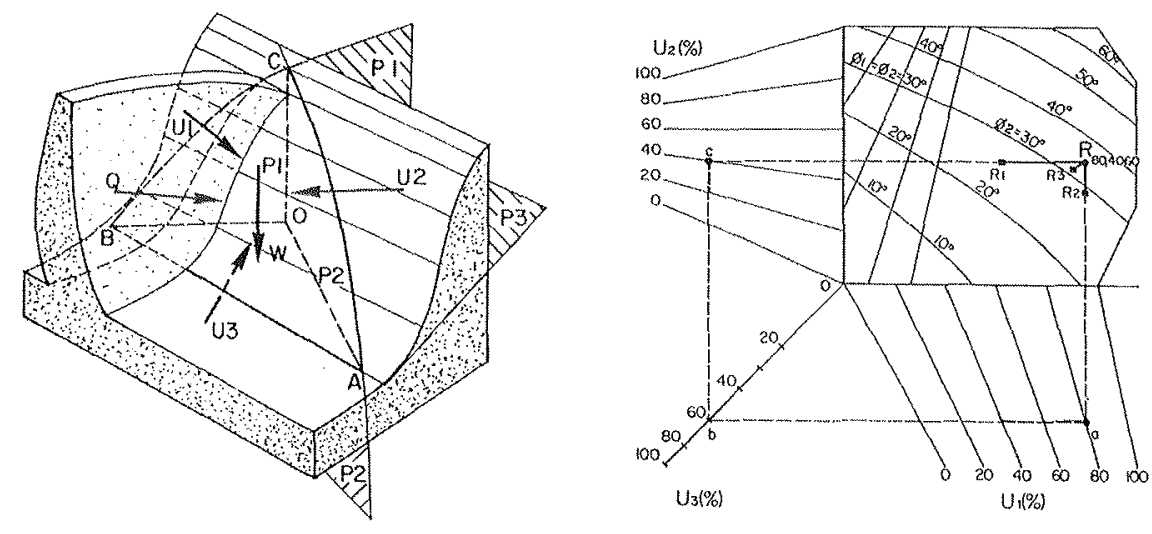

IG. 16. A gauche, définition des forces agissant sur un bloc tétraédrique
ABC : W poids, Q poussée de l'eau du réservoir, U1, U2, U3 sous-
pressions sur les faces P1, P2, P3; à droite, abaque graduée en angle
de frottement sur permettant de discuter les frottements et les sous
pressions sur chacune des trois faces internes (Londe, 1965).
Left, forces acting on a tetrahedral block ABC : W weight, Q thrust from
reservoir water; U1, U2, U3 uplift pressures on faces P1, P2, P3; right abacus
graduated along friction angles permitting to discuss friction and uplift upon
each of three faces (Londe, 1965).

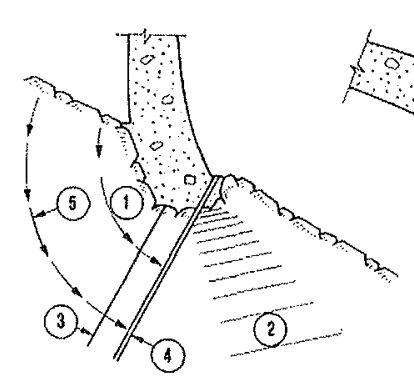

(a)

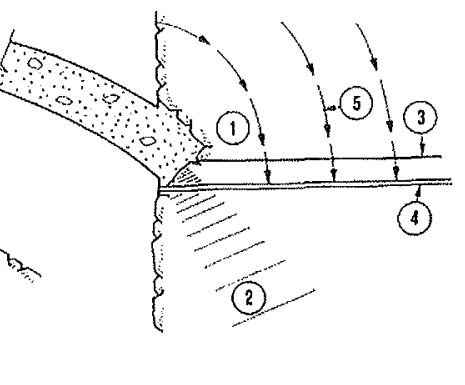

(b)

na. 17 Positions respectives recommandées du voile de drainage (4) et du voile d'injection (3), d'après Londe 1993, (a) en élévation et (b) en plan ; (1) désigne la zone en extension à l'amont immédiat de la fondation, (2) barrage interne créé par la compression, (5) les lignes de courant de l'écoulement/ autour du barrage.

Respective positions recommended for drain curtain (4) and grout curtain (3), after Londe 1993 (a) vertical and (b) horizontal cross sections; (1) designates the extension zone immediately upstream of dam founclation, (2) internal dam caused by compression, (5) flow lines around the dam foundation.

remplacés (le Gage, Ardèche), exploités à un niveau très bas (Beauregard, en Italie) ou abandonnés (Piney, Loire; Bromme, Cantal). Avant de succomber à la maladie qui le minait, André Coyne a procédé à une révision de toutes les voûtes en construction et en projet, et il a laissé à Pierre Londe l'obligation de comprendre pourquoi, pour la première fois au monde, une voûte pas particulièrement audacieuse avait pu tromper la confiance de ses constructeurs. Sa disparition dans l'année 1960 a conduit le tribunal à inculper l'ingénieur du Génie rural du Var, et elle l'a relaxé cinq ans plus tard en admettant avec la grande majorité des 19 experts que les éléments qui ont conduit à la rupture étaient imprévisibles : la Nature avait tendu un double piège, sur les qualités d'une roche bien différente de celles des sites des autres régions de France, et sur la

structure des surfaces de discontinuités, un piège qui aurait très probablement trompé tous les spécialistes de l'époque; la sauvegarde du chantier du pont de l'autoroute face à une pluviosité exceptionnelle avait faussé la prise de décision; et l'organisation des services de l'État avait été défaillante du début à la fin puisque aucun contrôle sérieux ne s'exerçait alors sur les barrages non destinés à la production d'électricité.

La législation a été profondément modifiée à cet égard et un comité technique permanent est chargé depuis de contrôler tous les barrages de plus de $15 \mathrm{~m}$, du projet à l'exploitation.

\section{3.}

\section{Les suites scientifiques}

Les ingénieurs ont compris d'abord qu'il fallait apporter davantage d'attention aux surfaces de discontinuité des massifs rocheux et ils ont longuement discuté les avantages et inconvénients respectifs des dispositifs destinés à l'étanchéité et au drainage des massifs de fondation : le drainage n'était systématique qu'au pied des barrages poids; il a été ajouté à maints barrages voûtes en service, souvent après des mesures qui montraient la réalité de sous-pressions dans le terrain à l'aval du barrage; il convient de le placer le plus à l'amont possible, parfois à partir d'une galerie intérieure à la base d'une voûte (Fig. 17). Ce (c voile ) de drainage justifié par la stabilité de l'ouvrage a pris davantage d'importance que le voile d'étanchéité classique, destiné à limiter les fuites du réservoir. Pour l'exploitant il implique surveillance et entretien car l'évolution naturelle des drains est souvent le colmatage. Rappelons qu'il n'y avait à Malpasset, pas plus que sur les barrages-voûtes d'avant 1960, aucun drain ni piézomètre en fondation; les concepts même de sous-pression et de voile de drainage n'étaient pas appliqués aux appuis des barrages-voûtes à cette époque.

C'est naturellement la communauté des ingénieurs en barrages qui a réagi la première au niveau international, la CIGB, Commission internationale des grands 
barrages, dont le congrès de 1964 à Édimbourg apparaît comme un premier congrès de mécanique des roches hors des mines, en posant sa question 28, "Propriétés physiques et mécaniques du rocher in situ; procéclés permettant de déterminer ces propriétés et de les améliorer, sous l'angle particulier de l'établissement du projet et de la construction des grands barrages m, question découlant évidemment de la rupture des fondations de Malpasset (les questions étant définies deux ans à l'avance, le congrès précédent en 1961 à Rome $n^{\prime}$ avait pas eu le recul nécessaire) : les comptes rendus de ce congrès permettent de constater que la mesure de la déformabilité s'est répandue largement dans le monde, surtout semble-t-il sur des ouvrages très importants (Kurobe IV au Japon, Dez en Iran, par exemple). Les essais au vérin, les mesures en forages, et les mesures sismiques sont abondamment cités et comparés.

Entre-temps l'écroulement du mont Toc dans la retenue du barrage italien de Vajont (9 octobre 1963) avait d'ailleurs ravivé l'urgence d'une approche à l'échelle du massif rocheux. En France, à l'initiative de D. Olivier Martin, directeur de l'Équipement à EDF (la branche qui construisait les grands écuipements, dont les barrages), le Comité français des grands barrages a mis en place quatre groupes de travail chargés des rapports français 15 à 18 de la question 28, respectivement sur la déformabilité du massif rocheux, les mesures mécaniques en forages, les effets physico-chimiques de l'eau, et la contribution des injections aux qualités mécaniques des massifs rocheux. Les congrès suivants ont maintenu le thème des fondations parmi les questions posées, notamment ceux d'Istanbul en 1967 et Montréal en 1970. La CIGB a lancé une enquête mondiale sur les incidents et accidents de barrages, publiée en 1973, et a demandé à Pierre Londe de rédiger une synthèse sur la mécanique des roches et les barrages (publiée en 1973, puis augmentée et mise à jour en 1993).

Plus souples dans leur programmation, les colloques annuels de géomécanique de Salzbourg avaient mis les barrages à leur programme dès 1962, en posant les bases d'une Société internationale alors qu'en
France l'Association nationale pour la recherche technique, ANRT, réunissait, à partir de 1963, les ingénieurs de génie civil avec des mineurs et des pétroliers dans une Commission de mécanique des roches, qui préfigurait le Comité national français, enregistré à la fin de 1967 (un an donc après le premier congrès international à Lisbonne). Parallèlement, les géologues vont convaincre la Délégation à la recherche scientifique et technique de financer des opérations concertées entre laboratoires, qui apporteront une contribution décisive à la mécanique des roches (Collectif, 1969), et ils fondent à leur tour les associations nationales et internationales de Géologie de l'ingénieur. Partie de presque rien, la mécanique des roches hors des mines aura acquis une place de tout premier plan en une décennie, s'étendant des barrages aux ouvrages souterrains pérennes et à la stabilité des versants rocheux, stabilité toujours précaire.

\section{Note sur les ruptures de barrages-voûtes}

Plusieurs accidents ont frappé des barrages-voûtes, qui ne sont pas des ruptures totales de la voûte: on cite souvent Clearwater en Californie dont la voûte a survécu à une crue qui a emporté les ailes en remblais ajoutées lors d'une augmentation de hauteur; on cite Vajont dans les Dolomites (Italie) qui n'a perdu que la route qui le couronnait alors qu'il a été surmonté par une vague de plus de $100 \mathrm{~m}$ lors de la chute d'un pan de montagne dans la retenue. Ces exemples, le second surtout, sont au contraire de remarquables témoignages de la capacité de résistance des structures en voûte, pourvu que les appuis supportent la poussée. On cite aussi des barrages modestes en Chine construits suivant un modèle qui a été rapidement abandonné. Ces cas ne mettent donc pas en défaut le titre de cet article.

\section{REMerelenGms}

En sus de l'appui constant de Pierre Habib, qui présente séparément les essais de laboratoire sur les gneiss de Malpasset, l'auteur a bénéficié de l'expérience de Jean-Louis Bordes, historien des techniques en qénéral et des techniques des barrages en particulier, et de celle de Bernard Goguel, spécialiste de la surveillance des barrages. 


\section{Bibliographie}

Références générales sur les barrages

Antoine P., Giraud A., Londe P. - Apport do la géologie structurale à la caractérisation géotechnique des fondations des grands ouvrages, in Les fondations des grands ouvrages, Coll. UNESCO, Mém. SGF 157, 1991, 112 p.

Carrère A. - Les leçons de Malpasset, leur application aux projets d'aujourd'hui. Revue française de géotechnique, $n^{\circ} 131$ 132, 2010.

Collectif - Lessons from dam incidents, bilingue, CIGB-ICOLD, Paris, 1973 $1100 \mathrm{p}$

Coyne A. - Cours de barrages. École nationale des ponts et chaussées, manuscrit polycopié, 1943-1959.

Leonards G.A. (sous la dir. de) - Dam failures (Coll. Purdue Univ., 1982), Eng. Geol., 24, 1987, p. 1-4.

Mary M. - Barrages-voûtes - Historique accidents et incidents. Dunod, Paris, 1968.

Références particulières sur le barrage de Malpasset

Bellier J. - Le barrage de Malpasset. Travaux, juillet $1967,20 \mathrm{p}$.

Bernaix J. - Étude géotechnique de la roche de Malpasset. Dunod, Paris, 1967.

Commission d'enquête (administrative) Le Génie civil, 1960, p. 255-257.

Commission judiciaire d'expertise - Le Génie civil, 1965, p. 239-246 et p. 277 . 283.

Commission judiciaire de contre-expertise - Le Génic civil, 1966, p. 14-20 et p. 99-108.

Dargeou J. - Le barrage de Malpasset. Travaux, 1955
Duffaut P. - Cinquantenaire de la rupture du barrage de Malpasset. Bulletin du Comité français d'histoire el de géologie, 2010. Sous presse.

Goguel J. - Rapport géologicue Malpasset Revue française de géotechnique, $n^{\circ} 131$ 132, 2010.

Habib P - La fissuration des gneiss de Malpasset. Revue française de géotechnique, $n^{\circ} 131-132,2010$.

Habib P. - The Malpasset Dam Failure, in Leonards G.A. Editor, Elsevier, 1987, p. 331-338.

Londe P. - The Malpasset Dam Failure, in Leonards G.A. Editor, Elsevier, 1987, p. 296-329

Post G., Bonazzi - Latest thinkings on the Malpasset accident, in Leonards G.A. Edition, Elsevier, 1987, p. 339-353.

Principales études de mécanique des roches concernant des barrages

Collectif-Catalogue des caractéristiques géologiques et mécaniques de quelques roches francaises (DGRST), L.CPC, Paris, 1969.

Drouhin G. - Essais géotechniques des terrains de fondation. $2^{e}$ Cong. intern. Grands barrages, CIGB, Paris, 1936.

Groupe de travail CFGB - La déformabilité des massifs rocheux, analyse et comparaison des résultats. $8^{c}$ Cong. intern. Grands barrages, Édimbourg, CIGB, Paris, 1964, I, p. 287-312.

Habib P. - Détermination des modules d'élasticité des roches en place. Annales de l'TTBTP 145, sept. 1950, p. 27-35.

Londe P. - Une méthode d'analyse à trois dimensions de la stabilité d'une rive rocheuse. Annales des Ponts et Chaussées, 135 (1), 1965, p. 37-60.
Londe P. - La mécanique des roches et les fondations des grands barrages, CIGB, Paris, 1973, mis à jour avec Le May Y., 1993, Rock foundations for dams, Bull, 88, CIGB, Paris.

Londe P., Sabarly F. - La distribution des perméabilités dans la fondation des barrages-voûtes en fonction du champ de

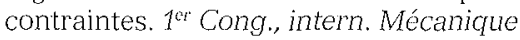
des roches, Lisbonne, 8-6 1966, p. 517521.

Louis C. - Etude des écoulements d'eau dans les roches fissurées et de leur influence sur la stabilité des massifs rocheux. Bull. DER (EDF) série A, 3, 1968, p. 5-132.

Louis C. - Introduction à l'hydraulique des roches. Bull. BRGM, 2" série 4, 1974, p. 283-356.

Maury V. - Mécanique des milieux stratifiés, Dunod, Paris.

Morlier P. - Le fluage des roches, Ann. ITBTP, 217, 1966, p. 91-111.

Rutqvist J., Stephansson O. - The Role of Hydromechanical Coupling in Fractured Rock Engineering. Hydrogeology Journal, 1st dec. 2002

Sabarly F - Les injections et les drainages des fondations de barrages. Géotechnique, 18, 1968, p. 229-249.

Schneider B. - Moyens nouveaux de reconnaissance des massifs rocheux. Annales de I'TTBTP, p. 235-236, p. 1055-1093.

Talobre J. - La mécanique des roches, Dunod, Paris, 1957 ( $2^{\mathrm{e}}$ éd. mise à jour en 1966). 